\title{
OPTIMIZING LINEAR EXTENSIONS
}

\author{
BRIDGET EILEEN TENNER
}

\begin{abstract}
The minimum number of elements needed for a poset to have exactly $n$ linear extensions is at most $2 \sqrt{n}$. In a special case, the bound can be improved to $\sqrt{n}$.
\end{abstract}

\section{INTRODUCTION AND DEFINITIONS}

A partially ordered set, or poset, $P=(X, \preceq)$ consists of a set $X$ together with a partial ordering $\preceq$ on $X$. For background on these structures, the reader is encouraged to review [3] and [4.

One statistic that can hint at how much information is missing in a partial ordering is based on the following definition.

Definition 1.1. A linear extension of a poset $P=(X, \preceq)$ is a total ordering of the elements of $X$ that is compatible with $\preceq$. The number of linear extensions of $P$ is denoted $e(P)$.

As suggested in [3], the number of linear extensions of a poset gives an indication of the intricacy of the original partial ordering. Thus understanding the function $e$ can provide some insight into the complexity of the structure of partial orderings.

Another poset statistic, the number of order ideals in a poset, is considered in [1], and a bound is given for the minimal number of elements needed to have a particular number of order ideals. Here, the analogous question is answered for the function $e$.

Definition 1.2. The size of a poset $P=(X, \preceq)$, denoted $|P|$, is the cardinality of $|X|$.

Definition 1.3. For any integer $n \geq 1$, set $\lambda(n)=\min \{|P|: e(P)=n\}$.

The main result of this work, Theorem 3.2 , is the bound

$$
\lambda(n) \leq 2 \sqrt{n} .
$$

In a certain case, as discussed in Section 4 , this bound can be improved further to $\sqrt{n}$. As displayed in Table 1, there are values of $n$ for which $\lambda(n)$ equals $2 \sqrt{n}$.

In the next section, the values of $\lambda(n)$ for small $n$ are given, together with examples of the posets that obtain them. Furthermore, the poset operations that give the primary tools for proving Theorem 3.2 are stated. Section 3 consists of the main result, and a special case is treated in the last section.

\section{EXAmples AND ARITHMetic of POSET OPERATIONS}

Before describing how basic poset operations affect the function $\lambda$, it is instructive to calculate $\lambda(n)$ for some small values of $n$, and to view the posets that give these values. These examples appear in Table 1, and as sequence A160371 in [2].

2000 Mathematics Subject Classification. Primary 06A07; Secondary 05A99, 06A05.

Key words and phrases. poset, linear extension, optimization. 


\begin{tabular}{|c|c|c|c|c|c|c|c|c|c|c|c|c|}
\hline$n$ & 1 & 2 & 3 & 4 & 5 & 6 & 7 & 8 & 9 & 10 & 11 & 12 \\
\hline$\lambda(n)$ & 0 & 2 & 3 & 4 & 4 & 3 & 5 & 4 & 5 & 5 & 5 & 4 \\
\hline $\begin{array}{c}\text { poset } \\
\text { example }\end{array}$ & $\emptyset$ & - . & - . & & & •.. & & ๖ & i & & & . \\
\hline$\lfloor 2 \sqrt{n}\rfloor$ & 2 & 2 & 3 & 4 & 4 & 4 & 5 & 5 & 6 & 6 & 6 & 6 \\
\hline
\end{tabular}

TABLE 1 . The values of $\lambda(n)$ for $1 \leq n \leq 12$, together with demonstrative posets, and the upper bound of Theorem 3.2 .

Two elementary operations on posets are the direct sum and the ordinal sum. Note that a poset which can be constructed entirely by these two operations is called series-parallel.

Definition 2.1. Let $P$ and $Q$ be posets on the sets $X_{P}$ and $X_{Q}$, respectively, with order relations $\preceq_{P}$ and $\preceq_{Q}$, respectively. The direct sum $P+Q$ is the poset defined on $X_{P} \cup X_{Q}$, with order relations $\preceq_{P} \cup \preceq_{Q}$. The ordinal sum $P \oplus Q$ is the poset defined on $X_{P} \cup X_{Q}$, with order relations $\preceq_{P} \cup \preceq_{Q} \cup\left\{x_{P} \preceq x_{Q}: x_{P} \in X_{P}, x_{Q} \in X_{Q}\right\}$.

The next lemma follows immediately from the definitions.

Lemma 2.2. For posets $P$ and $Q$,

$$
e(P+Q)=\left(\begin{array}{c}
|P|+|Q| \\
|P|
\end{array}\right) e(P) e(Q)
$$

and

$$
e(P \oplus Q)=e(P) e(Q) .
$$

Definition 2.3. For any $\ell \geq 0$, let the poset $C_{\ell}$ be the chain of $\ell$ elements, where $C_{0}=\emptyset$.

Certainly the poset $C_{\ell}$ is already a total ordering, so $\lambda\left(C_{\ell}\right)=1$ for all $\ell$. Moreover, it follows from the identities of Lemma 2.2 that

$$
e\left(P+C_{\ell}\right)=\left(\begin{array}{c}
|P|+\ell \\
|P|
\end{array}\right) e(P)
$$

and

$$
e\left(P \oplus C_{\ell}\right)=e(P)
$$

for all $\ell \geq 0$. Equation (11) implies that a poset with $n$ linear extensions can have arbitrarily large size. Perhaps unexpectedly, equation (11) will be very helpful in bounding $\lambda(n)$. The key is to employ it as in the following result.

Proposition 2.4. For all $\ell \geq 0$, $e\left(\left(P \oplus C_{\ell}\right)+C_{1}\right)=(|P|+\ell+1) e(P)$.

Proposition 2.4 gives the following initial result for all $n$.

Corollary 2.5. If $n=a b$ for $a, b \in \mathbb{Z}^{+}$with $a<b$, then $\lambda(n) \leq b$.

Proof. First note that

$$
\lambda(n) \leq n
$$

for all $n \in \mathbb{Z}^{+}$, by considering the $n$-element poset $C_{n-1}+C_{1}$, which has $n$ linear extensions. 


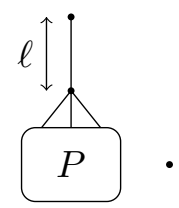

Figure 1. The poset $\left(P \oplus C_{\ell}\right)+C_{1}$ described in Proposition 2.4.

Let $P$ be a poset of size $\lambda(a)$, with $e(P)=a$. Since $a<b$, equation (2) implies $\lambda(a)<b$, and so $b-1-|P| \geq 0$. Set $Q=\left(P \oplus C_{b-1-|P|}\right)+C_{1}$. Then $|Q|=|P|+b-1-|P|+1=b$, and $e(Q)=(|P|+b-1-|P|+1) e(P)=a b=n$.

\section{BOUNDS}

The proof of the main result, Theorem [3.2, begins with an analysis of the following $m$-element poset $Q_{i, j, m}$, where $1 \leq i<j \leq m-2$. Note that $Q_{i, j, m}$ is not series-parallel.

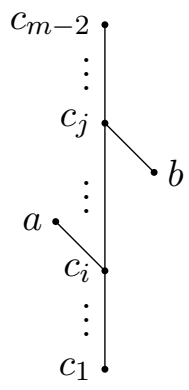

In any linear extension of $Q_{i, j, m}$, the elements $\left\{c_{1}, c_{2}, \ldots, c_{m-2}\right\}$ may appear in exactly one order. The element $a$ can appear anywhere after $c_{i}$, while the element $b$ can appear anywhere before $c_{j}$. The elements $a$ and $b$ are incomparable in $Q_{i, j, m}$, so they can appear in either order if they both appear between $c_{k}$ and $c_{k+1}$ in a linear extension. Thus

$$
e\left(Q_{i, j, m}\right)=(m-1-i) j+(j-i)=(m-i) j-i,
$$

and so

$$
\lambda((m-i) j-i) \leq m .
$$

Proposition 3.1. For all integers $n \geq 1$ and $d \geq 1$,

$$
\lambda(n) \leq\lfloor n / d\rfloor+d .
$$

Proof. This is proved by induction on $d$, where the case $d=1$ follows from equation (2).

Now suppose that $d \geq 2$ and that the result holds for all $d^{\prime} \in[1, d)$. The integer $n$ can be written as $n=q d-r$, where $r \in[0, d-1]$. If $r \geq 1$ and $q+r-2 \geq d$, then $Q_{r, d, q+r}$ is a poset having $n$ linear extensions and size

$$
q+r \leq\lfloor n / d\rfloor+1+(d-1)=\lfloor n / d\rfloor+d .
$$

Thus it remains to consider when $r=0$ or $q+r-2<d$.

If $r=0$, then $n=q d$ and Lemma 2.2 implies that

$$
\lambda(n) \leq \lambda(q)+\lambda(d) \leq q+d=\lfloor n / d\rfloor+d .
$$


This leaves the case when $r \in[1, d-1]$ and $q+r-1 \leq d$. The few cases that remain when $d \in\{2,3\}$ are easy to check (in fact, they concern only $n \leq 12$, and so appear in Table 1). For the conclusion of the argument, suppose $d \geq 4$.

Rewrite $n$ as $n=q^{\prime}(d-1)+r^{\prime}$ where $r^{\prime} \in[0, d-2]$. Because $n=q(d-1)+q+r$, the restrictions on $q, r$, and $d$ imply that there is at most one extra factor of $d-1$ in $q+r$. That is, $q^{\prime} \in\{q, q+1\}$. From the induction hypothesis for $d^{\prime}=d-1$, it follows that $\lambda(n) \leq q^{\prime}+d-1 \leq q+d$, which completes the proof.

Although the bound in Proposition 3.1 is linear, the fact that it holds for all integers $d \geq 1$ indicates that it can be improved further.

Theorem 3.2. For all $n \geq 1, \lambda(n) \leq 2 \sqrt{n}$.

Proof. Apply Proposition 3.1 with $d=\lceil\sqrt{n}$ and $\varepsilon=\lceil\sqrt{n}\rceil-\sqrt{n}$, where $\varepsilon \in[0,1)$ :

$$
\lambda(n) \leq\left\lfloor\frac{n}{\lceil\sqrt{n}\rceil}\right\rfloor+\lceil\sqrt{n}\rceil=\left\lfloor\sqrt{n}-\varepsilon+\frac{\varepsilon^{2}}{\sqrt{n}+\varepsilon}\right\rfloor+\sqrt{n}+\varepsilon .
$$

If $\varepsilon=0$, then $d=\sqrt{n}$, and the theorem holds. If $\varepsilon \in(0, .5]$, then $\varepsilon-1 \leq-\varepsilon$, and

$$
\left\lfloor\sqrt{n}-\varepsilon+\frac{\varepsilon^{2}}{\sqrt{n}+\varepsilon}\right\rfloor \leq\lfloor\sqrt{n}\rfloor=\sqrt{n}+\varepsilon-1 \leq \sqrt{n}-\varepsilon .
$$

On the other hand, if $\varepsilon \in(.5,1)$, then $\varepsilon-2<-\varepsilon$, and

$$
\left\lfloor\sqrt{n}-\varepsilon+\frac{\varepsilon^{2}}{\sqrt{n}+\varepsilon}\right\rfloor<\left\lfloor\sqrt{n}-\varepsilon+\frac{1}{2}\right\rfloor \leq\lfloor\sqrt{n}\rfloor .
$$

In other words, if $\varepsilon \in(.5,1)$, then

$$
\left\lfloor\sqrt{n}-\varepsilon+\frac{\varepsilon^{2}}{\sqrt{n}+\varepsilon}\right\rfloor \leq\lfloor\sqrt{n}\rfloor-1=\sqrt{n}+\varepsilon-2<\sqrt{n}-\varepsilon .
$$

Therefore, for any $\varepsilon \in[0,1)$, it follows from inequality (3) that $\lambda(n) \leq 2 \sqrt{n}$.

\section{A special CASE}

As suggested in Corollary 2.5, the number $\lambda(n)$ is influenced by the factorization of $n$. In particular, primality of $n$ can be a challenge for the function $\lambda$. On the other hand, if $n$ factors in a particular way, then the bound on $\lambda(n)$ can be further tightened along the lines of Corollary 2.5.

Corollary 4.1. If $n=a b$ for $a, b \in \mathbb{Z}^{+}$with $2 \sqrt{b}<a \leq b$, then $\lambda(n) \leq \sqrt{n}$.

Proof. Suppose that $n=a b$, where $1 \leq a \leq b<(a / 2)^{2}$. Construct a poset $P$ with $e(P)=b$ and $|P|=\lambda(b) \leq 2 \sqrt{b}<a$. Let $Q=\left(P \oplus C_{a-1-|P|}\right)+C_{1}$. Note that $e(Q)=a b=n$ and $|Q|=a$. Since $n=a b$ and $a \leq b$, this implies that $|Q| \leq \sqrt{n}$, and so $\lambda(n) \leq \sqrt{n}$.

\section{REFERENCES}

[1] K. Ragnarsson and B. E. Tenner, Obtainable sizes of finite topologies, to appear in J. Combin. Theory, Series A.

[2] N. J. A. Sloane, The on-line encyclopedia of integer sequences, published electronically at http: //www.research.att.com/ ${ }^{\sim}$ jas/sequences/.

[3] R. P. Stanley, Enumerative Combinatorics, vol. 1, Cambridge Studies in Advanced Mathematics, no. 49, Cambridge University Press, Cambridge, 1997. 
[4] W. T. Trotter, Combinatorics and Partially Ordered Sets: Dimension Theory, Johns Hopkins University Press, Baltimore, 1992.

E-mail address: bridget@math.depaul.edu

Department of Mathematical Sciences, DePaul University, Chicago, Illinois 60614 